Protiva Biotherapeutics of Burnaby, British Columbia in Canada, for its stable nucleic acid lipid particles (SNALPs). These are specialized lipid nanoparticles that encapsulate siRNA and were the first non-viral siRNA delivery vehicles that showed activity in non-human primates ${ }^{3}$. Alnylam has also launched a number of academic and industrial delivery collaborations (see 'The vehicle laboratory').

The rapid advancement of RNAi-based therapeutics is leading Polyplus-transfections to explore the manufacturing aspects of delivery vehicles. The company develops and markets DNA, RNA and protein transfection and delivery reagents for both in vitro and in vivo applications. And with RNAi therapeutics on the cusp of entering the clinic, the company sees the need to produce delivery vehicles in bulk under governmental quality specifications or 'current good manufacturing practices' (cGMP) standards. "If you want to be able to get these into the clinics, you have to have cGMP-qualified delivery systems," says Erbacher.

\section{Beyond the liver}

Despite this progress in general delivery, there is still some way to go for full therapeutic application. "I do not think that we are at the point now where we can name a tissue, specifically deliver and get good knockdown," says Anderson. Although he notes that there is good evidence to suggest that targeted systemic delivery should be possible.

Kim agrees that targeted delivery looks promising, but he cautions that the development of the delivery vehicles will be complicated. "You need a number of additional steps in the formulation process," he says.

A promising approach to targeted delivery involves incorporating targeting elements

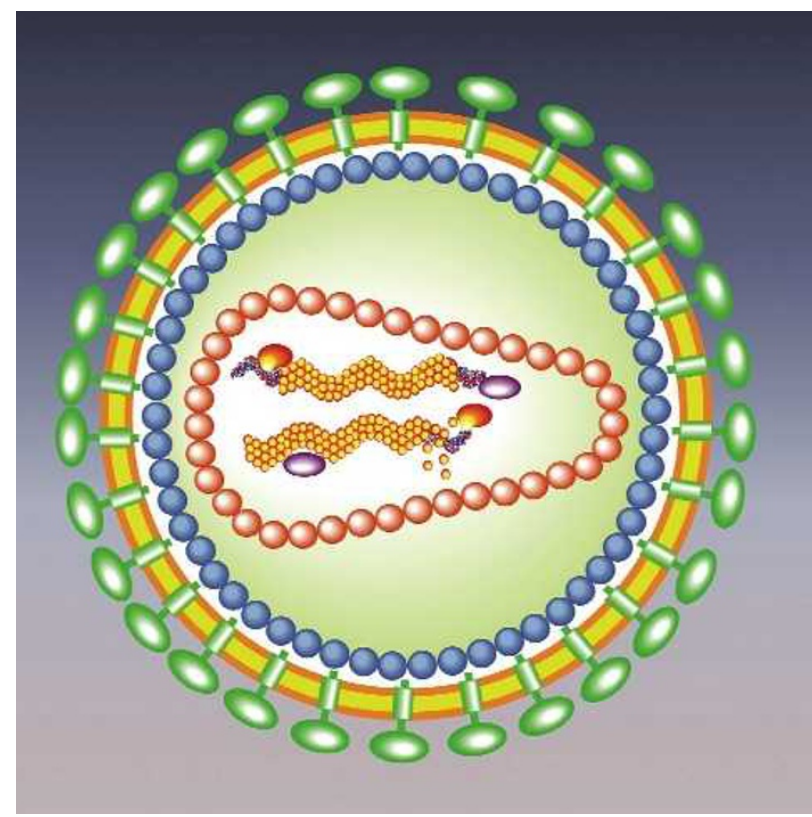

Lentiviruses, shown here schematically, can be modified to carry RNA into cells for potential therapeutic effect. such as antibody fragments, ligands and small chemical groups with the synthetic RNA. At the same time, techniques are being designed to image targeted nanoparticle delivery and biodistribution (see 'The inside track'). Some of the new approaches might overcome issues associated with lipid-based vehicles. For example, RNA aptamers attached to siRNAs, which have been used by researchers to target prostate cancer cells, might not cause the inflammatory responses seen in some cases with lipids or antibodies.

Another approach that might avoid inflammatory responses is being developed by Calando Pharmaceuticals in Pasadena, California, using polymers that contain cyclodextrin. When the polymers are mixed with siRNAs, they bind to the RNA backbone and assemble into nanoparticles. Targeting ligands or even stabilizing agents can then be attached to the cyclodextrin in the polymer to improve delivery.

\section{The evolutionary advantage}

Another approach to targeted delivery takes advantage of nature. "Viruses have learned how to get into cells - cross the membrane into the cytoplasm then get into the nucleus," says Inder Verma, a geneticist at the Salk Institute for Biological Studies in La Jolla, California. "All other approaches have to figure out how to do this." This gives viruses an "evo-

\title{
THINKING SMALL
}

"Each day you see major, significant publications on the roles of microRNAs in regulating pathways of genes involved in disease aetiology," says John Maraganore of Alnylam Pharmaceuticals in Cambridge, Massachusetts. Alongside the research into the mechanisms and roles of microRNA (miRNA) in disease, researchers are now starting to look at the potential of miRNA-based therapeutics.

Regulus Therapeutics in Carlsbad, California, is one of the first companies to be founded entirely for the development of miRNA-based therapeutics. It was formed in September as a joint venture between Alnylam and Isis Pharmaceuticals in Carlsbad. "We recognized that miRNAs were becoming a potential therapeutic opportunity," says Frank Bennett, senior vice-president of research at Isis, "but we also recognized that it was difficult for both companies to input the resources that were warranted." After much discussion, the two firms agreed to supply miRNA assets and core technologies to Regulus.

Regulus is taking two approaches to the development of miRNAbased therapeutics. "The most advanced approach is inhibiting the function of an endogenous miRNA in cells," says Bennett. This uses a synthetic oligonucleotide to

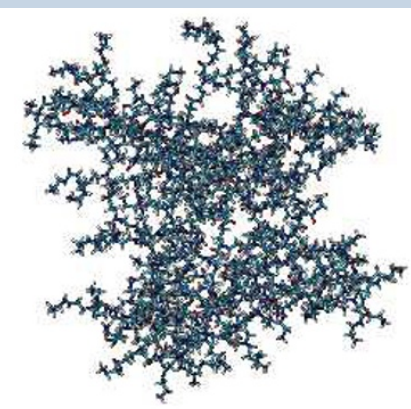

Testing of technologies, such as dendrimers, for delivering siRNAs may pave the way for miRNA drugs. target the miRNA for silencing. Although this is different from siRNA, as the miRNA is the target not the therapeutic agent, Bennett thinks that much of the technology developed for targeting mRNAs with siRNA is directly translatable to the targeting of miRNAs with oligonucleotides. And he notes that using oligonucleotides will also minimize any 'off-target' effects. "These oligonucleotides are very specific - even a single base mismatch will cause the oligo to lose activity," he says.

The second approach involves replacing miRNAs in or delivering them to cells, which Bennett says is similar to the current approach for siRNAs. The first applications here might be replacing miRNAs that are missing from diseaseassociated cells but present in a normal cells, which happens in some cancers, or augmenting naturally occurring miRNAs.

Although experience of siRNAs will help this nascent field, there is one unique issue in miRNA biology that researchers will have to address: miRNAs can inhibit tens to hundreds of genes at a time. Scores of researchers, both inside and outside academia, are now working to understand exactly how this regulation by miRNAs occurs. "You can use specific chemical modifications to the miRNA to limit that potential," says Bennett, "but it is still present."

Many researchers and companies now think miRNA-based therapeutics hold great promise for the future. And although some companies are waiting in the wings for more information on their basic mechanisms of action, others including Merck, Santaris Pharma of Hørsholm, Denmark, Rosetta Genomics in Rehovot, Israel, and Actigenics of MaurensScopont, France, are actively involved in miRNA research and development. 